\section{Oral Presentation}

\section{Methodology}

\section{EXPOSURE-LAG-RESPONSE IN OCCUPATIONAL EPIDEMIOLOGY: APPLICATION OF DISTRIBUTED NON- LINEAR LAG MODELS IN A COHORT OF DIATOMACEOUS EARTH WORKERS EXPOSED TO CRYSTALLINE SILICA}

\begin{abstract}
${ }^{1}$ Andreas Neophytou*, 'Sally Picciotto, 'Daniel Brown, ${ }^{1}$ Ellen Eisen, ${ }^{2}$ Harvey Checkoway, ${ }^{1}$ Sadie Costello. ${ }^{1}$ Division of Environmental Health Sciences, School of Public Health, University of California, Berkeley, Berkeley, CA, USA; ${ }^{2}$ Department of Family and Preventive Medicine, University of California, San Diego, San Diego, CA, USA
\end{abstract}

\subsection{6/oemed-2017-104636.108}

Occupational exposures extending over a long working life can have complex relationships with health outcomes, as timing, duration, and intensity of exposure are all potentially relevant. Simple measures of cumulative, or average intensity of exposure typically considered in occupational studies may not fully capture these relationships. We applied distributed nonlinear lag models to examine the association of crystalline silica exposures with mortality from lung cancer and nonmalignant respiratory disease. We fitted Cox proportional hazard models for each cause of interest to data from a cohort study of 2342 California diatomaceous earth workers exposed to crystalline silica. Our models combined various functions for exposure-response and lag-response including linear, piecewise constant and spline functions. Models with a spline function for exposure-response and a constant term for the lagresponse appeared to have the best fit for lung cancer, while models with spline functions for both exposure-response and lag-response had the best fit for non-malignant respiratory disease. Hazard ratios (HR) from these best fitting models corresponding to average daily exposures of $275 \mu \mathrm{g} / \mathrm{m} 3$ during lag years 11-40 prior to the age of observed cases were 1.96 (95\% confidence interval (CI) $0.95-4.06$ ) and 2.01 (95\% CI: 1.02-3.97) for the two outcomes respectively. HRs from simple models with linear exposure-response and constant lagresponse terms for the same exposure scenario were 1.15 (95\% CI: 0.88-1.49) and 1.21 (95\% CI: 1.01-1.44) respectively. Occupational studies of longitudinal cohorts with detailed exposure histories could benefit from methods allowing for non-linearities and the disentanglement of intensity, duration and timing of exposure.

\section{Oral Presentation}

\section{Risk Assessment}

\section{POOLED URANIUM MINERS ANALYSIS: GOALS AND CHALLENGES}

${ }^{1}$ David Richardson*, ${ }^{2}$ Michaela Kreuzer, ${ }^{2}$ Nora Fenske, ${ }^{2}$ Christina Sobotzki, ${ }^{3}$ Minh Do, ${ }^{3}$ Paul Demers, ${ }^{4}$ Dominique Laurier, ${ }^{4}$ Estelle Rage, ${ }^{5}$ Mary Schubauer-Berigan, ${ }^{6}$ Ladislav Tomasek, ${ }^{7}$ Jon Samet, ${ }^{8}$ Lydia Zablotska. ${ }^{1}$ University of North Carolina, Chapel Hill, NC, USA; ${ }^{2}$ Federal Office for Radiation Protection, Neuherberg, Germany; ${ }^{3}$ Cancer Care Ontario, Toronto, Canada; ${ }^{4}$ Institute for Radiation Protection and Nuclear Safety, Fontenayaux-Roses, France; ${ }^{5}$ National Institute for Occupational Safety and Health, Cincinnati, $\mathrm{OH}$, USA; ${ }^{6}$ Radiation Protection Institute, Prague, Czech Republic; ${ }^{7}$ University of Southern California, Los Angeles, CA, USA; ${ }^{8}$ University of California, San Francisco, San Francisco, CA, USA

\subsection{6/oemed-2017-104636.109}

Epidemiological studies of uranium miners helped to establish radon as a human carcinogen. However, radon remains a leading occupational cause of cancer mortality, and many workers are exposed to radon at levels at which excess risk of lung cancer has been observed in occupational cohort studies. Prior pooled studies of underground miners provided important quantitative estimates of radon-associated lung cancer risk. Recently, efforts have been undertaken to strengthen uranium miner studies to address contemporary occupational safety concerns. New cohorts of underground miners have been enumerated, existing cohorts have been expanded, and followup of the major cohorts of miners has been extended substantially. An international collaborative study has been undertaken to combine information from many of the world's most informative cohort studies of uranium miners; the combined study cohort encompasses more than 100000 miners. This talk will describe the major themes of this project, the goals of the collaborators and the challenges that we have encountered todate. We will describe similarities and differences between findings from these key cohorts and identify some major gaps in current knowledge about radon's effects on human health. Finally, we will discuss how international collaborative studies can strengthen our understanding of risks associated with occupational and environmental radon exposures. 


\section{Poster Presentation}

\section{Cancer}

\section{BREAST CANCER INCIDENCE AND METALWORKING FLUID EXPOSURE IN A COHORT OF FEMALE AUTOWORKERS}

${ }^{1}$ Erika Garcia*, ${ }^{2}$ Patrick Bradshaw, ${ }^{1}$ Ellen Eisen. ${ }^{1}$ Environmental Health Sciences Division, School of Public Health, University of California, Berkeley, Berkeley, California, USA; ${ }^{2}$ Epidemiology Division, School of Public Health, University of California at Berkeley, Berkeley, California, USA

\subsection{6/oemed-2017-104636.110}

Breast cancer is the leading cancer diagnosed among women and environmental studies have produced few leads on modifiable risk factors. Following an Institute of Medicine recommendation for occupational studies of highly exposed women, we took advantage of an existing cohort of 4503 female hourly autoworkers exposed to metalworking fluid (MWF), complex mixtures of oils and chemicals widely used in metal manufacturing worldwide. Cox proportional hazards models were fit to estimate hazard ratios (HR) for incident breast cancer and cumulative exposure (20 year lag) to straight mineral oils (a known human carcinogen), and water-based soluble and synthetic MWF. Because the state cancer registry began in 1985, decades after the cohort was defined, we restricted analyses to sub-cohorts hired closer to the start of cancer follow-up. Among those hired after 1969, the HR associated with an increase of one interquartile range in straight MWF exposure was 1.13 (95\% confidence interval: $1.03,1.23)$. In separate analyses of premenopausal breast cancer, as defined by age at diagnosis, the HR was elevated for exposure to synthetic MWF, chemical lubricants with no oil content, suggesting a different mechanism for the younger cases. This study adds to the limited literature regarding quantitative chemical exposures and breast cancer risk.

\section{Oral Presentation}

\section{Specific Occupations}

\section{LONGER TERM PHYSICAL HEALTH AND WELLBEING IN AUSTRALIAN GULF WAR VETERANS, 20 YEARS AFTER DEPLOYMENT}

Helen Kelsall*, Jillian Ikin, Stella Gwini, Andrew Forbes, Malcolm Sim. School of Public Health and Preventive Medicine, Monash University, Melbourne, Victoria, Australia

\subsection{6/oemed-2017-104636.111}

Background Ten years after the 1990-1991 Gulf War (GW), Australian veterans were found to have significantly poorer psychological health and some indicators of poorer physical health.

Methods A cohort of GW veterans and matched military comparison group were assessed at baseline (2000-2002) and follow-up (2011-2012), including a 63-item symptom checklist, modified CDC definition of multisymptom illness (MSI), doctor-diagnosed medical conditions since 2001, chronic fatigue and neurological symptom questionnaires. Additional measures e.g. irritable bowel syndrome (IBS) were included at follow-up.
Results From baseline, 715/1,330 veterans (54\%) and 675/ 1,449 comparison group (47\%) participated at follow-up. Relative to comparison group, GW veterans reported a higher average number of symptoms (ratio of means 1.36, 95\% CI 1.24-1.48), higher prevalence of MSI (risk ratio RR 1.60; 1.31-1.95), chronic fatigue RR 1.41 (1.02-1.96), IBS RR 1.64 (1.18-2.27) and 6/40 medical conditions. GW veterans were significantly more likely to report $\geq 1$ RR 1.13 (1.03-1.25) or $\geq 4$ RR 1.32 (1.07-1.64) neuropathic symptoms. From baseline to follow-up, overall, symptom prevalence and MSI increased and remained higher in GW veterans; the gap between GW veterans' and comparison group symptomatology remained unchanged; chronic fatigue prevalence more than doubled in both groups, and there was a non-significantly greater incidence of chronic fatigue in GW veterans.

Conclusions These finding indicate enduring increased health symptoms and longer term adverse physical health outcomes associated with GW service, and highlight the importance of effective detection and management of chronic physical conditions and improved awareness among health practitioners of conditions occurring more commonly in veterans.

\section{Poster Presentation}

\section{Exposure Assessment}

\section{A 10 YEAR PROSPECTIVE STUDY OF TONER HANDLING WORKERS}

Ryosuke Sugano*, Satoshi Michii, Hajime Ando, Hiroki Nozawa, Kazunori Ikegami, Akira Ogami. Department of Work Systems and Health, Institute of Industrial and Ecological Sciences, University of Occupational and Environmental Health, Kitakyushu, Fukuoka, Japan

\subsection{6/oemed-2017-104636.112}

Purpose To measure the risk of pulmonary disease due to toner dust exposure, in a 10 year prospective cohort study among toner handling workers.

Methods Subjects that were included in the analysis of this study were 260 male employees of a Japanese photocopier, printer and toner production company. Onset of pneumoconiosis, pulmonary fibrosis, granulomatous pneumonia and lung cancer were assumed as endpoints of the investigation, and blood markers (KL-6,SP-D), respiratory function index and the chest CT shadow reading were used as substitute end-points for before the onset of these diseases. Disease onset was determined via a self-administered questionnaire, blood tests and respiratory function tests were conducted once a year, while Chest CT examinations were conducted in the $1 \mathrm{st}$, 5th and 10 th year of the study. Subjects were classified by duration of toner handling work into four groups - long-exposure (>20 years): $n=65$ (mean 40 years, smoking rate $35 \%$ ); medium-exposure (10-20 years): $n=71$ (mean age 31.9 years, smoking rate $47.9 \%)$, and short-exposure ( $<10$ years): $n=50$ (mean age 31.6 years, smoking rate $46 \%$ ). The average dust levels in the environment of the toner handling work decreased well below the ACGIH allowable concentrations through the period of the study.

Results None of the endpoint diseases developed in any of the four groups. Annual percent change for blood marker and respiratory function levels, and chest CT parameters were compared across the four groups but no statistical significance was seen. 